\title{
DOMINANT STRATEGY IMPLEMENTATION IN MULTI-UNIT ALLOCATION PROBLEMS
}

\author{
MANIPUSHPAK MITRA AND ARUNAVA SEN
}

\begin{abstract}
In this paper we analyze allocation problems where an efficient rule can be implemented in dominant strategies with balanced transfers. We first prove an impossibility result in the homogenous goods case when preferences over these goods are allowed to be sufficiently diverse. We then consider a package assignment problem where the planner can bundle or package various units of the homogenous goods and wishes to allocate the packages efficiently. We characterize the package schemes for which an efficient rule in the associated package assignment problem can be implemented in dominant strategies with balanced transfers.
\end{abstract}

\section{INTRODUCTION}

In this paper we consider two allocation problems and analyze the possibility of identifying domains of preferences over which efficient outcomes can be implemented in dominant strategies with balanced transfers. Preferences of the players or agents are assumed to be quasi-linear and their valuations for the commodities are assumed to be private information. The objective of the planner is to design a mechanism that attains the following:

(1) each agent has dominant strategy incentives to reveal the truth and

(2) the outcome in every state of the world is efficient.

The former requires that truthful reporting is a dominant strategy of all agents under all profiles or states of the world. The latter requires the allocation to maximize the sum of utilities it generates and also for aggregate transfers to be balanced.

Although the requirements above are stringent, there are important theoretical reasons for investigating environments and allocation problems where they can be satisfied. Some of these reasons are elucidated in Section 1.1 and a more complete discussion can be found in Mitra and Sen [12]. An example of an allocation problem and an environment where all the objectives can be reconciled is the single machine sequencing problem with linear costs, first analyzed in Suijs [13]. The model and results were generalized in Mitra [10], [11].

Our objective in this paper is to extend this line of research to another familiar class of allocation problems, that of allocating $m$ homogenous indivisible commodities amongst $n$ agents. For instance, the commodities could be identical plots of land and the agents could be farmers. Each farmer can receive and (possibly) has use for more than one plot of land. These valuations, however are private information. Agents can be compensated by

Date: April 9, 2009. 
money and utility functions are quasi-linear. Efficiency requires the $m$ units to be allocated in a way which maximizes the sum of agent utilities from the commodities. Moreover transfers must be zero in the aggregate. The question we address is the following: does there exist a reasonable restriction on agent valuations so that efficiency can be attained with dominant strategy incentives for agents to reveal their valuations? Our result in this case is negative: we show that these requirements are mutually incompatible on any domain that satisfies a mild richness condition.

In view of our negative result, we analyze a variant of the problem above where the planner can bundle or package various units and wishes to allocate these packages in a fully efficient way in dominant strategies. If there are $n$ agents and $m$ units, a package scheme is an $n$ vector $\left(q_{1} \leq q_{2} \leq \ldots \leq q_{n}\right)$ with $q_{1}+q_{2}+\ldots+q_{n}=m$. Note that efficiency in this context is weaker than standard efficiency. For instance, suppose that $n=3, m=6$ and the package scheme is the vector $(1,2,3)$. Here the planner is constrained to give 3 units to one agent, 2 to another and 1 to the third while standard efficiency may require all 6 units to be given to one agent. We characterize package schemes which have the property that there exists some admissible, nontrivial domain over which it can be implemented efficiently with balanced transfers. ${ }^{1}$ We can show that the scheme $(1,2,3)$ can be implemented in the sense above in the $n=3, m=6$ case and is indeed, the only one with this property.

1.1. Related Literature. In the mechanism design literature an important result is that in the quasi-linear setting, the class of Vickrey-Clarke-Groves (or VCG) mechanisms (Vickrey [15], Clarke [1] and Groves [3]) achieves truth telling in dominant strategies and guarantees an efficient allocation in every state. Moreover if domain of valuation is convex the the VCG mechanisms are the only ones that have these properties (Holmström [6]). ${ }^{2}$ In our problem we assume that our domain is convex which implies smooth connectedness. Hence, in our framework too, VCG mechanisms are the only mechanisms that works.

The main difficulty with VCG mechanisms is that in typical domains they are not budget balancing (Groves and Ledyard [4], Green and Laffont [2], Hurwicz [7], Hurwicz and Walker [8] and Walker [16]). The failure to obtain balanced VCG mechanisms is quite serious since under these circumstances, the social optimum in the second-best sense may not require getting the decision on the allocation exactly correct in terms of efficiency. There are a number of papers such as Groves and Loeb [5], Tian [14] and Liu and Tian [9] which have investigated the structure of pure public goods problems where full efficiency can be attained with dominant strategies. Results in the same spirit for sequencing problems have been established by Suijs [13]. There is therefore a compelling reason to investigate domains on which VCG mechanisms "work".

\footnotetext{
${ }^{1}$ Efficiency here is, of course, with respect to the given scheme.

${ }^{2}$ Holmström [6] showed that if a domain is "smoothly connected" then we have the uniqueness of VCG mechanisms. Since convex domains are smoothly connected, uniqueness of VCG mechanisms also follow when the domain is convex.
} 


\section{Homogenous Goods Problem: An Impossibility Result}

We now consider the problem of allocating $m$ identical units of an object amongst $n$ agents. The main result is that there are no "non-trivial" domains over which an efficient rule can be implemented by balanced transfers.

Let $N=\{1,2, \ldots, n\}$ denote the finite set of $n$ agents. Let $m$ denote the number of identical indivisible units of a given commodity to be allocated to these $n$ agents. Let $\theta_{j}(k) \in \Re_{+}$represent the utility of the $j^{\text {th }}$ agent if she receives $k$ units where $k \in\{0,1,2, \ldots, m\}$. The vector $\theta_{j}=\left(\theta_{j}(1), \ldots, \theta_{j}(m)\right) \in \Re_{+}^{m}$ represents the type of agent $j$. We make two basic assumptions regarding types.

(1) $\theta(k+1) \geq \theta(k)$ for all $k=1, \ldots, m-1$, i.e. receiving more units is no worse than not receiving them.

(2) $\theta_{j}(0)=0$, i.e. the utility of receiving no units is normalized to zero.

The domain of type vectors of agent $j$ is denoted by $\Theta \subseteq \Re_{+}^{m}$. A state is a set of $n$ vectors $\theta=\left(\theta_{1}, \ldots, \theta_{n}\right) \in \Theta^{n}$. An allocation is a vector of non-negative integers $x=\left(x_{1}, \ldots, x_{n}\right)$ such that $x_{j} \in\{0,1,2, \ldots, m\}$ and $\sum_{i \in N} x_{i}=m$. Let $X$ denote the set of all possible allocations. Given an allocation $x=\left(x_{1}, \ldots, x_{n}\right) \in X$, the utility of an agent $j$ with type $\theta_{j} \in \Theta$ is $U_{j}\left(x_{j}, t_{j} ; \theta_{j}\right)=\theta_{j}\left(x_{j}\right)+t_{j}$ where $t_{j} \in \Re$ is the transfer that she receives. A multi-unit allocation problem $\Gamma$ is a triple $\langle N, m, \Theta\rangle$.

Definition 2.1. An allocation $x^{*} \in X$ is efficient for state $\theta \in \Theta^{n}$ if $x^{*} \in$ $\arg \max _{x \in X} \sum_{j \in N} \theta_{j}\left(x_{j}\right)$.

An efficient rule (also denoted by $x$ ) associates an efficient allocation with every state $\theta \in \Theta^{n}$. The main objective of the planner is to ensure an efficient allocation in every profile. The difficulty however is that agents have private information about their valuations. The planner therefore has to design a mechanism to induce the agents to reveal their private information. It is well known that by applying the Revelation Principle we can concentrate on direct revelation mechanism where agents report their types and, based on their reports, the planner decides (i) an allocation of the $m$ goods and (ii) a transfer for each agent. Formally, a (direct) mechanism $M$ is a pair $\langle x, t\rangle$, where $x \in X$ and $t \equiv\left(t_{1}, \ldots, t_{n}\right): \Theta^{n} \rightarrow R^{n}$. If $M=\langle x, t\rangle$ is the mechanism, then an announcement $\hat{\theta}=\left(\hat{\theta}_{1}, \ldots, \hat{\theta}_{n}\right) \in \Theta^{n}$, results in agent $j$ of type $\theta_{j}$ getting utility $U_{j}\left(x_{j}(\hat{\theta}), t_{j}(\hat{\theta}) ; \theta_{j}\right)=\theta_{j}\left(x_{j}(\hat{\theta})\right)+t_{j}(\hat{\theta})$.

Definition 2.2. An efficient rule $x^{*}: \Theta^{n} \rightarrow X$ for $\Gamma=\langle N, m, \Theta\rangle$ is implementable, if there exists a mechanism $M=\left\langle x^{*}, t\right\rangle$ such that, for all $j \in N$, for all $\theta_{j}, \theta_{j}^{\prime} \in \Theta$ and for all $\hat{\theta}_{-j} \in \Theta^{n-1}$, we have

$$
U_{j}\left(x_{j}^{*}\left(\theta_{j}, \hat{\theta}_{-j}\right), t_{j}\left(\theta_{j}, \hat{\theta}_{-j}\right) ; \theta_{j}\right) \geq U_{j}\left(x_{j}^{*}\left(\theta_{j}^{\prime}, \hat{\theta}_{-j}\right), t_{j}\left(\theta_{j}^{\prime}, \hat{\theta}_{-j}\right) ; \theta_{j}\right) .
$$

In other words the mechanism induces each agent to reveal their type truthfully independent of what they believe about the announcements and true types of the other agents. It is obvious that when agents are truthful, an efficient allocation is achieved. In addition to the requirements above, we impose budget balancedness. 
Definition 2.3. An efficient rule $x^{*}$ in $\Gamma=\langle N, m, \Theta\rangle$ is implementable with balanced transfers if there exists a mechanism $M=\left\langle x^{*}, t\right\rangle$ that implements it and furthermore $\sum_{j \in N} t_{j}(\theta)=0$ for all $\theta \in \Theta^{n}$.

Thus, an efficient rule $x^{*}$ is implementable with balanced transfers if it can be implemented in a manner such that aggregate transfers are zero in every state. In such problems, incomplete information does not impose any welfare loss as the transfers are within the agents. Our goal is to identify problems $\Gamma=\langle N, m, \Theta\rangle$ which have the property that there exists an efficient rule which can be implemented by balanced transfers. In order to do so, we introduce a minimal richness requirement on domains.

Definition 2.4. The domain $\Theta$ is minimally rich if it satisfies the following conditions:

(1) There exists $\alpha, \beta \in \Theta$ such that $\alpha(k)>\beta(k) \geq 0$ for all integers $k \in\{1,2, \ldots, m\}$ and $\alpha(m)>\alpha(m-r)+r \sum_{p=0}^{r} \beta(p)$ for all $r \in$ $\{1,2, \ldots, m\}$.

(2) $\Theta$ is convex, that is if $\alpha, \beta \in \Theta$ then $\lambda \alpha+(1-\lambda) \beta \in \Theta$ for all $\lambda \in[0,1]$.

The first part of the minimal richness assumption guarantees the existence of two sufficiently "diverse" type vectors. The vector $\alpha$ must strictly dominate the vector $\beta$. Moreover the $m^{\text {th }}$ or last component of the $\alpha$ must be strictly greater than the sum of the $r^{\text {th }}$ component of $\alpha$ and $r$ times the sum of the first $r$ components of $\beta$. Observe that it is satisfied if the vector $(0,0, \ldots, 0)$ and any other strictly positive vector exists in the domain. In fact for any $\alpha$ with distinct components, we can satisfy the condition if we can pick another feasible type vector which is sufficiently smaller than $\alpha$ componentwise. In this sense we can say that the condition is satisfied if we can pick two type vectors, one of which is sufficiently larger than the other.

Why do we impose an assumption such as (1) and why do we think that it is appropriate to refer to it as a requirement of non-triviality? The following example clarifies these issues.

Example 2.5. Let $n=m$ and $\bar{\Theta}=\{\lambda \alpha+(1-\lambda) \beta: \forall \lambda \in[0,1]\}$ where $\alpha \equiv(\alpha(1), \ldots, \alpha(n))=(a, \ldots, a), \beta=(\beta(1), \ldots, \beta(n))=(b, \ldots, b)$ and $a>b>0$. In other words, each agent has zero marginal utility for units in excess of one. The domain fails to satisfy minimal richness because $\alpha(n)=a$ and $\alpha(n-1)+\beta(1)=a+b$ implies that $\alpha(n)<\alpha(n-1)+\beta(1) .{ }^{3}$ The domain is such that all efficient rules allocate exactly one unit to all agents in every state, that is $x^{*}(\theta)=\left(x_{1}^{*}(\theta)=1, \ldots, x_{n}^{*}(\theta)=1\right)$ for all $\theta \in \bar{\Theta}$. Clearly there are no incentive problems and the efficient rule can be implemented with no transfers (no announcements are required either).

Minimal richness "forces" the efficient rule to have some variation across states. In particular, for every agent $j$, it guarantees the existence of a state where $j$ receives all $m$ units. The example makes it clear that without an assumption of this sort, implementability with balanced transfers may be satisfied trivially. We can now present our general impossibility theorem.

\footnotetext{
${ }^{3}$ This condition is a violation of condition (1) of minimal richness for $m=n$ and $r=1$.
} 
Theorem 2.6. Let $\Gamma=\langle N, m, \Theta\rangle$ be a multi-unit allocation problem where $\Theta$ is minimally admissible. Then $\Gamma$ cannot be implemented with balanced transfers.

Proof: Let $\Gamma=\langle N, m, \Theta\rangle$ be a multi-unit allocation problem where $\Theta$ is minimally admissible and let $x^{*}$ be an efficient rule in $\Gamma$. Since the domain is convex and transfers are balanced, the results of Holmström [6] and Walker [16] can be applied to infer that the implementing mechanism can be assumed w.l.o.g to be a VCG mechanism and that $x^{*}$ must satisfy the following condition: For all pairs of profiles $\theta, \theta^{\prime} \in \Theta^{n}$, we must have

$$
\sum_{S \subseteq N}(-1)^{|S|} \sum_{i \in N} \theta_{i}\left(x_{i}^{*}(\theta(S))\right)=0
$$

where $\theta(S)=\left(\theta_{1}(S), \ldots, \theta_{n}(S)\right) \in \Theta^{n}$ is a state such that $\theta_{j}(S)=\theta_{j}$ if $j \notin S$ and $\theta_{j}(S)=\theta_{j}^{\prime}$ if $j \in S$.

Let $\alpha, \beta$ be type vectors which satisfy condition (1) of minimal richness (i.e $\alpha(k)>\beta(k)$ for all $k \in M$ and $\alpha(m)>\alpha(m-r)+r \sum_{p=0}^{r} \beta(p)$ for all $r \in M)$. Consider a pair of states $\theta, \theta^{\prime} \in \Theta^{N}$ where $\theta=(\alpha, \ldots, \alpha)$ and $\theta^{\prime}=(\beta, \ldots, \beta)$. Given any $S \subseteq N, \theta(S)=\left(\theta_{1}(S), \ldots, \theta_{n}(S)\right) \in \Theta^{n}$ where $\theta_{j}(S)=\theta_{j}=\alpha$ if $j \notin S$ and $\theta_{j}(S)=\theta_{j}^{\prime}=\beta$ if $j \in S$. Our objective is to calculate the LHS of the expression in (2.1).

The pair $\theta$ and $\theta^{\prime}$ is selected in such a way that for any $S \subset N$, the efficient allocation $x^{*}(\theta(S))$ is one where all the units are allocated to agents in the set $N-S$, i.e. $x_{i}(\theta(S))=0$ for all $i \in S$. To see this consider any $S_{1} \subset N$ such that $\left|S_{1}\right|=n-1$. By setting $r=m$ in condition (1) of minimally richness it follows $\alpha(m) \geq m \sum_{p=0}^{m} \beta(p)$. This means that any efficient rule allocates all the $m$ units to $\left\{i_{1}\right\}=N-S_{1}$ in state $\theta\left(S_{1}\right)$ and hence $\theta_{i}\left(x_{i}^{*}\left(\theta\left(S_{1}\right)\right)=0\right.$ for all $i \in S_{1}$. Therefore, $\sum_{i \in N} \theta_{i}\left(x_{i}^{*}\left(\theta\left(S_{1}\right)\right)\right)=\alpha(m)$ for all $S_{1}$ such that $\left|S_{1}\right|=n-1$. Consider any $S_{2} \subset N$ such that $\left|S_{2}\right|=n-2$. Again, all agents with type $\beta$ (that is $i \in S_{2}$ ) gets nothing because $\alpha(m) \geq m \sum_{p=0}^{m} \beta(p)$. Hence, $\theta_{i}\left(x_{i}^{*}\left(\theta\left(S_{2}\right)\right)=0\right.$ for all $i \in S_{2}$. Moreover, since there are exactly two agents with type $\alpha$ in any state $\theta\left(S_{2}\right)$, the allocation for $\left\{i_{1}, i_{2}\right\} \in N-S_{2}$ is determined by that $k \in\{0,1,2, \ldots, m\}$ for which $\alpha(m-k)+\alpha(k)$ is maximized. Hence, $\sum_{i \in N} \theta_{i}\left(x_{i}^{*}\left(\theta\left(S_{2}\right)\right)\right)=\alpha\left(m-k^{*}\right)+\alpha\left(k^{*}\right) \geq \alpha(m)$ where $k^{*} \in\{0,1,2 \ldots, m\}$ maximizes $\alpha(m-k)+\alpha(k)$. Thus, $\sum_{i \in N} \theta_{i}\left(x_{i}^{*}\left(\theta\left(S_{2}\right)\right)\right)=$ $\alpha(m)+\epsilon_{1}$ where $\epsilon_{1}=\alpha\left(m-k^{*}\right)+\alpha\left(k^{*}\right)-\alpha(m) \geq 0$. Continuing this way we obtain:

Given any $h \in\{1, \ldots, n\}$, for all $S_{h} \subset N$ such that $\left|S_{h}\right|=n-h$,

$$
\sum_{i \in N} \theta_{i}\left(x_{i}^{*}\left(\theta\left(S_{h}\right)\right)\right)=\alpha(m)+\epsilon_{h-1}
$$

where $\epsilon_{n-1} \geq \ldots \geq \epsilon_{2} \geq \epsilon_{1}=\epsilon_{0}=0$.

An important observation at this point is that all the $\epsilon$ terms depend only on $\alpha$. Finally, if $S=N$ (that is, $\theta(N)=\theta^{\prime}$ ), we get

$$
\sum_{i \in N} \theta_{i}\left(x_{i}^{*}(\theta(N))\right)=\sum_{i \in N} \beta\left(x_{i}^{*}(\theta(N))\right)=\sum_{i \in N} \beta\left(x_{i}^{*}\left(\theta^{\prime}\right)\right)<\alpha(m)
$$


Substituting (2.2) and (2.3) in the left hand side of (2.1) and then simplifying it we get

(2.4)

$$
\sum_{S \subseteq N}(-1)^{|S|} \sum_{i \in N} \theta_{i}\left(x_{i}^{*}(\theta(S))\right)=\sum_{p=0}^{n-1}(-1)^{p}\left(\begin{array}{l}
n \\
p
\end{array}\right) \epsilon_{n-1-p}+(-1)^{n-1}\left(\alpha(m)-\sum_{i \in N} \beta\left(x_{i}^{*}\left(\theta^{\prime}\right)\right)\right)
$$

If the right hand side of (2.4) is not equal to zero then we already have a violation of (2.1). However, if the right hand side of (2.4) is zero then we consider a pair of states $\theta, \tilde{\theta} \in \Theta$ such that $\theta=\left(\theta_{1}=\alpha, \ldots, \theta_{n}=\alpha\right)$ and $\tilde{\theta}=\left(\tilde{\theta}_{1}=\tilde{\beta}, \ldots, \tilde{\theta}_{n}=\tilde{\beta}\right)$ where $\tilde{\beta}=\lambda \alpha+(1-\lambda) \beta$ and $\lambda \in(0,1)$. Selecting $\lambda>0$ sufficiently close to zero we get $\alpha(m)>\alpha(m-r)+r \sum_{p=0}^{r} \tilde{\beta}(p)$ for all $r \in\{1,2, \ldots, m\}$. Using the same arguments as before with the pair $\theta, \tilde{\theta}$ instead of the pair $\theta, \theta^{\prime}$ we get:

$$
\sum_{S \subseteq N}(-1)^{|S|} \sum_{i \in N} \theta_{i}\left(x_{i}^{*}(\theta(S))\right)=\sum_{p=0}^{n-1}(-1)^{p}\left(\begin{array}{l}
n \\
p
\end{array}\right) \epsilon_{n-1-p}+(-1)^{n-1}\left(\alpha(m)-\sum_{i \in N} \tilde{\beta}\left(x_{i}^{*}(\tilde{\theta})\right)\right)
$$

Since the $\epsilon$ terms in (2.5) are the same as those in (2.4) (because they depend only on $\alpha$ ), the only difference between (2.5) and (2.4) is the last sum on the right hand side. Given, $\alpha(k)>\beta(k)$ for all $k \in\{1,2, \ldots m\}$, and $\tilde{\beta}=\lambda \alpha+$ $(1-\lambda) \beta$, we get $\tilde{\beta}(k)>\beta(k)$ for all $k \in\{1,2, \ldots m\}$. Thus $\sum_{i \in N} \tilde{\beta}\left(x_{i}^{*}(\tilde{\theta})\right)>$ $\sum_{i \in N} \beta\left(x_{i}^{*}\left(\theta^{\prime}\right)\right)$ so that the RHS of $(2.5)$ is non-zero. Therefore, we have a violation of (2.1) which proves that the efficient rule cannot be implemented with balanced transfers.

\section{Packaging Problem: Possibility Results}

We have seen in the previous section that in the standard multi-unit allocation problem, it is impossible to implement an efficient rule with balanced transfers except in cases where the problem is virtually trivial. In this section we consider a variant of this problem and demonstrate some possibility results. We consider the problem where the planner can bundle or package various units and wishes to allocate these packages efficiently. Observe that packaging creates "partial" heterogeneity in the goods being allocated. We use the qualification "partial" in the statement above because we allow for cases where some of the packages are of the same size. We address the following question: does there exist a package scheme such that the efficient rule can be implemented with balanced transfers over some nontrivial domain? We show that the answer is affirmative for all package schemes except for some special cases. As part of the domain we also characterize the domain of utilities for which a package scheme is implementable with balanced transfers. We now proceed to details.

As before, we let $N=\{1, \ldots, n\}$ and $m$ denote the set of agents and number of (identical) goods respectively. A package scheme or simply, a scheme is a vector of $n$ integers $q=\left(q_{1}, \ldots, q_{n}\right)$ such that $q_{1} \leq q_{2} \leq \ldots \leq q_{n}$ with $\sum_{i=1}^{n} q_{i}=m$. For every scheme $q$, we let $\Sigma(q)$ be the set of all possible permutations of the components of the vector $q$. For any $q$, an allocation $x^{q}$ is an element of the set $\Sigma(q)$. We shall let $x_{j}^{q}$ denote the package assigned to agent $j$ under $x^{q}$. When the scheme $q$ being referred to is evident from the context, we suppress the superscript in $x^{q}$. 
We illustrate the notation above by reference to an example. Assume that the set of agents is $\{1,2,3\}$ and that $m=6$. Suppose that $q=(0,1,5)$. An allocation assigns 5 units to one agent, 1 to another. Suppose that $x^{q}$ gives 5 units to agent 2 , then $x_{2}^{q}=5$ and so on.

Fix a scheme $q$. A type for agent $j$, is a vector $\theta_{j}=\left(\theta_{j}\left(q_{1}\right), \ldots, \theta_{j}\left(q_{n}\right)\right) \in$ $\Re_{+}^{n}$ where $\theta_{j}\left(q_{k}\right)$ denotes the utility of receiving $q_{k}$ units for agent $j$. We shall let $\Theta^{q}$ denote the domain of such type vectors (assumed, once again, to be the same for all agents). Observe that since the components of the vector $q$ need not be distinct, there may be components of $\theta^{q}$ which are identical to each other. We assume

(1) If $q_{k}=0$ for some $k$, then $\theta\left(q_{k}\right)=0$

(2) If $q_{k}=q_{k+1}$ for some $k$, then $\theta\left(q_{k}\right)=\theta\left(q_{k+1}\right)$.

We shall let $\Theta^{q}$ denote the set of possible type vectors for the scheme $q$. For any scheme $q$, a package allocation problem is a triple $\Gamma^{q}=\left\langle N, m, \Theta^{q}\right\rangle$.

Definition 3.1. Consider a package problem $\Gamma^{q}=\left\langle N, m, \Theta^{q}\right\rangle$. An allocation $x^{*} \in \Sigma(q)$ is said to be $q$-efficient in state $\theta \in\left[\Theta^{q}\right]^{n}$ if

$$
x^{*} \in \arg \max _{x \in \Sigma(q)} \sum_{j \in N} \theta_{j}\left(x_{j}\right) .
$$

An allocation is $q$-efficient in a package problem $\Gamma^{q}$ if the various packages which constitute $q$ cannot be permuted amongst the agents to increase aggregate utility. Of course, an allocation which is q-efficient is not necessarily efficient because the argmax in its definition is only with respect to $\Sigma(q)$ rather than the union of $\Sigma(q)$ 's for all possible $q$ 's.

A $q$-efficient allocation rule is a mapping $x^{*}:\left[\Theta^{q}\right]^{n} \rightarrow \Sigma(q)$ which picks an allocation $x^{*}(\theta)$ which is efficient in state $\theta$ for all $\theta \in\left[\Theta^{q}\right]^{n}$. We say that the package problem $\Gamma^{q}=\left\langle N, m, \Theta^{q}\right\rangle$ is implementable if there exists a $q$-efficient rule $x^{*}$ and a mechanism $M=\left\langle x^{*}, t\right\rangle$ which induces each agent to reveal her type truthfully, i.e. $j \in N$, for all $\theta_{j}, \theta_{j}^{\prime} \in \Theta^{q}$ and for all $\hat{\theta}_{-j} \in\left[\Theta^{q}\right]^{n-1}$, we have

$$
\theta_{j}\left(x_{j}^{*}\left(\theta_{j}, \hat{\theta}_{-j}\right)\right)+t_{j}\left(\theta_{j}, \hat{\theta}_{-j}\right) \geq \theta_{j}\left(\left(x_{j}^{*}\left(\theta_{j}^{\prime}, \hat{\theta}_{-j}\right)\right)+t_{j}\left(\theta_{j}^{\prime}, \hat{\theta}_{-j}\right)\right.
$$

We say that the $\Gamma^{q}=\left\langle N, m, \Theta^{q}\right\rangle$ is implementable with balanced transfers if there exists a $q$-efficient rule and a mechanism $M=\left\langle x^{*}, t\right\rangle$ which implements it and furthermore $\sum_{j \in N} t_{j}(\theta)=0$ for all $\theta \in\left[\Theta^{q}\right]^{n}$.

We wish to address the following question: do schemes exist which can be implemented with balanced transfers over "non-trivial" domains? We are clearly motivated by the impossibility result of the previous section. Since efficiency with balanced transfers over non-trivial domains cannot be achieved, can the units be packaged according to some scheme $q$ such that a $q$-efficient rule can then be implemented with balanced transfers?

We let $\Delta \theta_{j}=\left(\Delta \theta_{j}\left(q_{1}\right), \ldots, \Delta \theta_{j}\left(q_{n-1}\right)\right)$ represent the vector of first differences generated by the vector $\theta_{j} \in \Theta^{q}$, i.e. $\Delta \theta_{j}\left(q_{k}\right)=\theta_{j}\left(q_{k+1}\right)-\theta_{j}\left(q_{k}\right)$ for all $k \in\{1, \ldots, n-1\}$. An important observation is that all difference vectors $\Delta \theta_{j}$ have non-negative components. Moreover, if $q_{k+1}=q_{k}$, then $\theta_{j}(k)=0$. For any domain $\Theta^{q}$, we denote its corresponding first difference domain $\Delta \Theta^{q}$. Finally, we say that $\Delta \theta_{j}<\Delta \theta_{j}^{\prime}$ if $\Delta \theta_{j}\left(q_{k}\right)<\Delta \theta_{j}^{\prime}\left(q_{k}\right)$ for all 
$k \in\{1, \ldots, n-1\}$ such that $q_{k+1}>q_{k}$. Note that unlike in the heterogenous goods case we cannot require one difference vector to strictly dominate another. This is because if $q_{k+1}=q_{k}$ for some $k$, then all difference vectors have their $k^{t h}$ component equal to zero.

Definition 3.2. The domain $\Delta \Theta^{q}$ satisfies regularity if for all $\Delta \gamma$ in the relative interior of $\Delta \Theta^{q}$, there exists $\Delta \alpha, \Delta \beta \in \Delta \Theta^{q}$ such that $\Delta \alpha<\Delta \gamma<$ $\Delta \beta$.

Definition 3.3. The domain $\Theta^{q}$ is admissible if it is a convex subset of $\Re^{n}$ satisfying regularity.

In the definition of an admissible domain, there is a "natural ordering" with respect to which these differences are computed. This is the ordering $\{1,2, \ldots, n\}$ which arises naturally because the components of the vector $q$ are arranged in ascending order and utilities are increasing in the number of units that an agent receives. We believe that the admissibility requirement is weak. Besides convexity, it imposes only regularity restrictions on admissible utility differences.

The main result in this section characterizes admissible domains over which a package scheme is implementable by balanced transfers.

Theorem 3.4. For any scheme $q$, let $\Gamma^{q}=\left\langle N, m, \Theta^{q}\right\rangle$ be a package problem where $\Theta^{q}$ is an admissible domain. Then $\Gamma^{q}$ is implementable by balanced transfers if and only if the associated difference domain is of the form $\Delta \Theta^{q}=$ $\left\{(1-s) . \delta+s . \delta^{\prime} \mid s \in I\right\}$ where $I \subset \Re_{+}$is an interval. Moreover if $I$ is nontrivial, $\delta, \delta^{\prime} \in \Re_{+}^{n-1}$ are such that (i) $\delta^{\prime}>\delta$ and (ii) $\sum_{k=1}^{n-1}(-1)^{k-1}\left(\begin{array}{c}n-2 \\ k-1\end{array}\right) \delta_{k}=$ $\sum_{k=1}^{n-1}(-1)^{k-1}\left(\begin{array}{l}n-2 \\ k-1\end{array}\right) \delta_{k}^{\prime}$.

The proof of the Theorem 3.4 is very similar to the proof of the main result in Mitra and Sen [12] and is hence omitted.

Theorem 3.4 states that if $\Gamma^{q}=\left\langle N, m, \Theta^{q}\right\rangle$ (where $\Theta^{q}$ is admissible) is implementable by balanced transfers, then the associated difference domain must be a straight line in $\Re_{+}^{n-1}$ satisfying certain restrictions. But for an arbitrary $q$ can one find an admissible $\Theta^{q}$ such that $\Gamma^{q}=\left\langle N, m, \Theta^{q}\right\rangle$ is implementable by balanced transfers? The answer is negative as the following example demonstrates.

Example 3.5. Let $n=3, m=4$ and $q=(1,1,2)$. A typical difference vector is of the form $(0, \lambda)$ where $\lambda>0$ is a real number. Let $\delta=(0, \lambda)$ and $\delta^{\prime}=\left(0, \lambda^{\prime}\right)$ be the two vectors specified in Theorem 3.4. Then $\lambda=$ $-\sum_{k=1}^{n-1}(-1)^{k-1}\left(\begin{array}{c}n-2 \\ k-1\end{array}\right) \delta_{k}=-\sum_{k=1}^{n-1}(-1)^{k-1}\left(\begin{array}{c}n-2 \\ k-1\end{array}\right) \delta_{k}^{\prime}=\lambda^{\prime}$. Therefore $\delta=\delta^{\prime}$ which contradicts the requirement that $\delta^{\prime}>\delta$.

Below we provide a complete answer to the question of what schemes are implementable with balanced transfers over some admissible domain. For any scheme $q$ let $\Delta q$ denote the $n-1$ vector $\left(\Delta q_{1}, \ldots, \Delta q_{n-1}\right)$ where $\Delta q_{k}=q_{k+1}-q_{k}$, for $k=1, \ldots, n-1$.

Theorem 3.6. Let $q$ be a scheme. There exists an admissible domain $\Theta^{q}$ such that $\Gamma^{q}=\left\langle N, m, \Theta^{q}\right\rangle$ is implementable by balanced transfers if and only if there exist integers $r, s \in\{1, \ldots, n-1\}$ such that $\Delta q_{r}, \Delta q_{s} \neq 0$ and $r+s$ is an odd integer. 
Proof: We first prove necessity. Suppose that $q$ is a scheme such that there exists an admissible domain $\Theta^{q}$ and $\Gamma^{q}=\left\langle N, m, \Theta^{q}\right\rangle$ is implementable with balanced transfers. According to Theorem 3.4 there must exist $n-1$ dimensional vectors $\delta$ and $\delta^{\prime}$ such that $\delta^{\prime}>\delta$ and $\sum_{k=1}^{n-1} \hat{\rho}_{k} \delta_{k}^{\prime}=\sum_{k=1}^{n-1} \hat{\rho}_{k} \delta_{k}$ where $\hat{\rho}_{k}=(-1)^{k-1}\left(\begin{array}{c}n-2 \\ k-1\end{array}\right)$. Therefore $\sum_{k=1}^{n-1} \hat{\rho}_{k}\left(\delta_{k}^{\prime}-\delta_{k}\right)=0$. Since $\delta^{\prime}>\delta$, $\delta_{k}^{\prime}-\delta_{k} \geq 0$ for all $k$ and strictly positive for at least one $k$. Observe that $\hat{\rho}_{k}$ is strictly positive for $k$ odd and strictly negative for $k$ even. Note also from the definition of the difference domain that $\delta_{k}^{\prime}$ and $\delta_{k}$ can be strictly positive only for those values of $k$ for which $\Delta q_{k}$ is strictly positive. Suppose that for all $r, s$ such that $\Delta q_{r}, \Delta q_{s}>0$, we have that $r+s$ is an even integer, i.e. all $k$ such that $\Delta q_{k}>0$ are even or all are odd. Clearly then $\sum_{k=1}^{n-1} \hat{\rho}_{k}\left(\delta_{k}^{\prime}-\delta_{k}\right)=0$ cannot hold and we obtain a contradiction to Theorem 3.4.

In order to prove sufficiency, let $r$ and $s$ be integers such that $\Delta q_{r}, \Delta q_{s}>$ 0 and $r+s$ is an odd integer. Let $\delta$ be the $n-1$ dimensional vector $(0,0, \ldots, 0)$. Pick $\epsilon>0$ and real numbers $c$ and $d$ and let $\delta^{\prime}$ be an $n-$ 1 dimensional vector where $\delta_{k}^{\prime}=0$ if $\Delta q_{k}=0, \delta_{r}^{\prime}=c, \delta_{s}^{\prime}=d$ and $\delta_{k}^{\prime}=\epsilon$ for all other $k$. Moreover $c$ and $d$ are picked to satisfy the equation $\hat{\rho}_{r} \cdot c+\hat{\rho}_{s} \cdot d+T=0$ where $T=\epsilon\left[\sum_{k \notin[Q \cup\{r\} \cup\{s\}]} \hat{\rho}_{k}\right]$ where $Q=$ $\left\{k \in\{1, \ldots, n-1\}-\{r, s\} \mid \Delta q_{k}=0\right\}$. Since $\hat{\rho}_{r}$ and $\hat{\rho}_{s}$ are integers and are of opposite sign we can find strictly positive $c$ and $d$ which satisfy the equation for any given $T$. We now construct a difference domain which is a segment of the line passing through $\delta$ and $\delta^{\prime}$. It can be easily verified that this domain satisfies the requirements specified in Theorem 3.4.

Theorem 3.6 makes it easy to check whether there exists an admissible domain over which a scheme can be implemented with balanced transfers. For instance, we have an impossibility result for all $q$ if $n=2$. In this case $\Delta q$ is a singleton so that there does not exist $r, s$ such that $\Delta q_{r} \neq \Delta q_{s}$. On the other hand if $n \geq 3$ and $q$ is a scheme such that all the components of $q$ are distinct, (for instance if $n=3, m=10$ and $q=(1,2,7)$ ), then we have a possibility result.

Finally consider the case where $m=k n$ for some positive integer $k$ and consider the scheme $q=(k, k, \ldots, k)$. Here all agents get $k$ units in every state. It is therefore trivially implementable with balanced transfers over any arbitrary domain which appears to contradict Theorem 3.4. However this is not so because the associated difference domain for any domain consists of the single vector, the origin in $\Re^{n-1}$. This is the case where the interval $I$ in Theorem 3.4 is trivial, i.e. consists of a single point.

\section{Conclusion}

In this paper we have first established an impossibility theorem in a homogenous goods allocation problem where the domain satisfies a minimal richness requirement. Given this impossibility we consider package assignment problems in the homogenous goods case. We obtained a characterization of package schemes that can be implemented in dominant strategies with balanced transfers. These results clearly suggest that one can find possibility results by introducing appropriate heterogeneity in the homogenous goods problem. 


\section{REFERENCES}

[1] Clarke, E.H., 1971. Multi-part pricing of public goods. Public Choice 11, 17-33.

[2] Green, J., Laffont, J.J., 1979. Incentives in Public Decision Making. North Holland Publication, Amsterdam.

[3] Groves, T., 1973. Incentives in teams. Econometrica 41, 617-631.

[4] Groves, T., Ledyard, J.O., 1977. Some limitations of demand revealing processes. Public Choice 29, 107-124.

[5] Groves, T., Loeb, M., 1975. Incentives and public inputs. Journal of Public Economics 4, 211-226.

[6] Holmström, B., 1979. Groves schemes on restricted domains. Econometrica 47, 11371144.

[7] Hurwicz L. 1975. On the existence of allocative systems whose manipulative Nash equilibria are Pareto optimal. Mimeo, University of Minnesota.

[8] Hurwicz, L., Walker, M., 1990. On the generic non-optimality of dominant strategy allocation mechanisms: A general theorem that includes pure exchange economies. Econometrica 58, 683-704.

[9] Liu, L., Tian, G., 1999. A characterization of the existence of optimal dominant strategy mechanisms. Review of Economic Design 4, 205-218.

[10] Mitra, M., 2001. Mechanism Design in Queueing Problems. Economic Theory 17(2), 277-305.

[11] Mitra, M., 2002. Achieving the First Best in Sequencing Problems. Review of Economic Design 7(1), 75-91.

[12] Mitra, M., Sen, A. 2008. Efficient Allocation of Heterogeneous Commodities with Balanced Transfers, mimeo.

[13] Suijs, J., 1996. On incentive compatibility and budget balancedness in public decision making. Economic Design 2, 193-209.

[14] Tian, G., 1996. On the existence of optimal truth-dominant mechanisms. Economics Letters 53, 17-24.

[15] Vickrey, W., 1961. Counterspeculation, auctions and competitive sealed tenders. Journal of Finance 16, 8-37.

[16] Walker, M., 1980. On the non-existence of dominant strategy mechanisms for making optimal public decisions. Econometrica 48, 1521-1540.

Economic Research Unit, Indian Statistical Institute, Kolkata, India.

E-mail address: mmitra@isical.ac.in

Planning Unit, Indian Statistical Institute, New Delhi, India.

E-mail address: asen@isid.ac.in 